\title{
Algebraic Computations
}

\section{Algebraic Relationships}

Algebraic relationships between variables or expressions are very common in computer algebra. Not only do they often occur explicitly, in forms like $\operatorname{SQRT}\left(\mathrm{X}^{2}+1\right)$, but well known difficulties such as $\sin (\mathrm{x})^{* * 2}+\cos (\mathrm{x})^{* *} 2=1$ (Stoutemyer, 1977) can be expressed in this form. Nevertheless it is difficult to compute with regard to these algebraic relationships. This chapter discusses the problem of such computing, and then enters the area of algebraic geometry, which is a natural outgrowth of attempts to perform such computations as readily as one computes without them.

The simplest case of computing subject to algebraic relationships consists of computations in the field $\{K(X, Y) \mid F(X, Y)=0\}$ where $F$ is a rational function (which can be reduced to a polynomial by multiplying out any denominator) in which $X$ and $Y$ appear effectively, and $K$ is a field of characteristic* 0 . As a simple example, I shall consider $F(X, Y)=Y^{2}-X^{2}+1$ as the definition of the algebraic relationship.

One of the key ideas in efficient computer algebra is that of canonical forms, by which is meant ensuring that equivalent expressions, however computed, are represented by equivalent data structures. This has the great merit that the case of unequal expressions is normally detected very early in any comparison operation. Since our computations are likely to tax the power of even the largest computers, this point is one we cannot afford to neglect.

The first problem that arises in the example is that $Y^{2}$ and $X^{2}-1$ are the same

* We will not normally (except in Chapter 8 ) be concerned with fields of finite characteristic, and any reference to a field should be construed as a reference to a field of characteristic 0 unless the converse is stated. 
function as a result of the relationship between $X$ and $Y$. In order to retain canonical representations of our algebraic expressions, we must perform some trick such as replacing $Y^{2}$ by $X^{2}-1$ wherever it occurs. This must be done at a very low level in all parts of the system, otherwise our algebra system will find that the matrix

$$
\left(\begin{array}{cc}
X-1 & Y \\
Y & X+1
\end{array}\right)
$$

has determinant $\left(X^{2}-1\right)-Y^{2}=0$ but rank 2 because the rank calculations did not apply this equivalence at the right stage. This makes it very difficult to add algebraic relationships to a system which does not include a good treatment of algebraic expressions and the relations to which they give rise. In particular the usual method of introducing square roots into REDUCE-2 (Hearn,1973) by means of a rule such as

FOR ALL X LET SQRT $(X)^{* * 2=X ;}$

will not work in similar complicated cases, because the transformation is not applied at the correct level.

Replacing high powers of $Y$ (in our example $Y^{2}$ ) absolutely everywhere can cause problems in some cases: consider attempting to take the g.c.d. of $X^{2}-X^{*} Y+1$ and $X^{*} Y$ by a standard Euclidean algorithm, with $X$ considered to be a more significant variable than $Y$, i.e. working in $K[Y][X]$. In the obvious notation, the procedure goes as follows:

$\begin{array}{lll}\text { Explanation } & U & V \\ \text { Initial state } & X^{* *} 2-Y^{*} X+1 & X^{*} Y \\ U:=U^{*} Y-V^{*} X & -X^{*} Y^{* *} 2+Y & X^{*} Y \\ & =-X^{* *} 3+X+Y & X^{*} Y \\ U:=U^{*} Y+V^{*} X^{* * 2} & X^{*} Y+Y^{* * 2} & \\ & =X^{* * 2}+X^{*} Y-1 & X^{*} Y \\ U:=U^{*} Y-V^{*} X & X^{*} Y^{* *} 2-Y & X^{*} Y \\ & =X^{* *} 3-X-Y & X^{*} Y \\ & & X^{*} Y\end{array}$

Continuing Loop 
More complex cases can be adduced to show that ordering $Y$ before $X$ does not always avoid these problems. In actual fact, since $X^{2}-1=Y^{2}=(X-1)^{*}(X+1)$, and $Y, X-1, X+1$ are all irreducible, $K[X, Y]$ is not a unique factorisation domain, so that we cannot successfully take g.c.d.s in the domain $\{K[X, Y] \mid F(X, Y)=0\}$. Any representation based on g.c.d.s will fail unless we have a very clear idea of precisely what we mean by a g.c.d., and what algebraic dependencies we are to consider while doing so.

This makes it more difficult to devise a canonical representation for expressions in $\{K(X, Y) \mid F(X, Y)=0\}$. In my REDUCE-2 programs* to perform algebraic-geometric computations I have adopted the following representation, which is based on restricting $F$ to be a polynomial in $X$ and $Y$, monic in $Y$. If $F$ is not monic, i.e. it has a leading coefficient $G$ which is not a unit in $K[X]$, then we can write $Y^{\prime}=G^{*} Y$, and then $Y^{\prime}$ satisfies a monic equation $F^{\prime}\left(X, Y^{\prime}\right)=0$ and $\{K(X, Y) \mid F(X, Y)=0\}$ is isomorphic to $\left\{K\left(X, Y^{\prime}\right) \mid\right.$ $\left.F^{\prime}\left(X, Y^{\prime}\right)=0\right\}$. Furthermore, in most algebra systems (and certainly in REDUCE-2) elements of a field $K$ are represented as the quotient of two elements of an integral domain $L$ (e.g. rationals are the quotient of two integers, or rational functions are the quotient of two polynomials). We then insist (for purely practical reasons) that all the coefficients of $F(X, Y)$ lie in $L$ (if not, multiply by all the denominators, and then deal with the nonmonicity of the equation as described above). This leads to the assertion that $L[X, Y]^{2} \leq L[X, Y]$. The technique of extending by non-monic polynomials is used by Cohen $\&$ Yun (1979) to introduce particular fractions into integral domains, but we do not require this, since we always assume that we have a field available to work in.

If these assumptions are satisfied, then an element of $\{K(X, Y) \mid F(X, Y)=0\}$ can be represented as $A / B$, where $B$ is in $K[X]$ and $A$ is in $\{K[X, Y] \mid F(X, Y)=0\}$ and $A$ and $B$ are coprime as elements of $K[X, Y]$ (i.e. ignoring the relation between $X$ and $Y$ ) and $B$ is normalised in some sense (e.g. leading coefficient of $B$ positive) to prevent one multiplying $A$ and $B$ by some element of $\mathrm{K}$ to obtain a different representation of the same element of $\{K(X, Y) \mid F(X, Y)=0\}$.

\footnotetext{
* Further details can be found in Appendix 1.
} 


\section{Uniqueness of Algebraics}

However, being able to manipulate algebraic expressions in the manner described above is not sufficient unless one has a guarantee that they are well-formed (in the sense that $f(X, Y)$, regarded as a polynomial in $Y$, has no roots in $K(X))$, for otherwise one has no guarantee of canonical representation even if one is using the system described in the previous paragraph. While $F(X, Y)=Y^{2}-X^{2}+2 X-1$ is a perfectly reasonable algebraic relationship, and defines an algebraic curve, the curve is not irreducible and the algebraic extension of $K(X)$ defined by that equation is in fact $K(X)$, since the equation factors into linear factors. Similar considerations are brought out in the integration algorithm (Risch,1970), where one has to express certain residues as elements of a $\boldsymbol{Z}$-module, and therefore has to recognise that $\sqrt{-1}$ and $\sqrt{-4}$ are linearly dependent over the integers*. Similarly, the correct algebraic-geometric model for the integration of

$$
\sqrt{x^{2}-1}+\frac{1}{\sqrt{1-1 / X^{2}}}
$$

is not $\left\{K(X, Y, Z) \mid Y^{2}-X^{2}+1=0 Z^{2}-1+1 / X^{2}=0\right\}$ with the integrand being $Y+1 / Z$, but $\left\{K(X, Y) \mid Y^{2}-X^{2}+1\right\}$ with the integrand being $Y+X / Y$.

In order to test that an algebraic expression is well-formed, we need to ensure that $F(X, Y)$ is an irreducible ${ }^{\$}$ polynomial (strictly speaking, has only one factor involving $Y$

* This is brought out forcibly in Chebyshev's integral (Appendix 2, Example 5), where, having discovered that SQRT(2) is an independent algebraic entity over $Q$, we then have to consider SQRT(4 SQRT(2) + 9). This is not in fact independent over $\boldsymbol{Q}(\operatorname{SQRT}(2))$, since it is $2 \operatorname{SQRT}(2)+1$.

\$ This glosses over one point. We need to ensure that it is absolutely irreducible (i.e. irreducible over the algebraic closure of the constant field). Now if $F(X, Y)$ is such a polynomial, choose a value $y$ of $Y$ such that $F(X, y)$ is square free (possible if $F(X, Y)$ is). Then if $F(X, Y)$ is absolutely irreducible, it is irreducible over the splitting field of $F(X, y)$, because any factorisation of $F(X, y)$ which corresponds to a factorisation of $F(X, Y)$ can be "grown up" to that factorisation without extending the constant domain (see any $p$-adic factorisation technique, e.g. Wang (1978) or Yun $(1973,1976)$ ). 
effectively: but any factor not involving $Y$ might as well be removed anyway). If $K$ is $Q$ (or a purely transcendental extension of $\boldsymbol{Q}$ ), then this is the problem of factorising multivariate polynomials over the integers, which is known to be soluble (Wang \& Rothschild,1975 and Yun,1976). If $K$ is not purely transcendental, then we need to use algorithms which have recently been developed (see Trager,1976) for factoring over algebraic fields. If we have purely radicals, rather than general algebraic expressions, then we could use many of the algorithms of Zippel (1977).

\section{Representations of Algebraics}

If we wish to deal with more than one algebraic expression, we have a choice: we can deal with both of them directly in a multivariate representation, or we can express them both in terms of one (more complicated) algebraic expression, viz a primitive element for the field (we will term this a primitive representation). The mathematical theory is nearly always cast in terms of primitive elements, since this makes the notation and descriptions easier. However primitive elements have their disadvantages: with them one would represent $\sqrt{2}+\sqrt{3}+\sqrt{5}+\sqrt{7}+\sqrt{11}$ as a polynomial of degree 31 in a primitive element which satisfied an equation of degree 32 . This can prove extremely expensive, and furthermore it must be converted back into the other form if it is going to make sense to a user. Also it may happen that a problem posed in terms of two algebraic expressions (e.g. $\left.\operatorname{SQRT}\left(\mathrm{X}^{* *} 2-1\right), \operatorname{SQRT}\left(\mathrm{X}^{* * 2} 2-2\right)\right)$ decomposes into two sub-problems, each of which involves only one of the algebraic expressions. In a system in which the two expressions are stored separately it is extremely easy to take advantage of this decomposition, while a primitive element system would find that very hard. For these reasons I use a multivariate expression rather than a primitive element expression, though this decision is largely a matter of opinion. This question was also considered by Caviness \& Rothstein (1976), who came to the same conclusion, but also without any hard theoretical evidence. It would be

\# See van der Waerden (1949), Vol I, pp. 126,127 (applying the fact that all fields of characteristic 0 are perfect, and hence have only separable extensions, from pp. 124125). 
interesting to produce such evidence. It is worth noting that the discussion in Chapter 7 accompanying the algorithm DENOMINATOR_ALGEBRAIC, which constructs a defining equation for an algebraic number in order to determine its denominator, is not really evidence for either side, since the same construction would still be necessary, and only marginally easier to program, in a primitive representation system.

If a multivariate representation is chosen, then a general field looks like $\{K(X, Y, Z) \mid F(X, Y)=0, G(X, Y, Z)=0\}$, where $F$ involves $\#$ and $Y$ effectively, and $G$ involves $Z$ and one of $X$ and $Y$ (but not necessarily both) effectively (there is a discussion of such representations by Shtokhamer(1977)). One example of this is $\{K(X, Y, Z) \mid$ $\left.Y^{2}-1-X^{2}=0, Z^{2}-2-X^{2}=0\right\}$. If we choose this representation, we need a way of deciding whether an algebraic expression is independent of the previous ones, i.e. a mechanism for factoring polynomials over multivariate algebraic fields. This can be obtained from the work of Trager(1976), whose algorithm ALG_FACTOR (see also under Algorithm ALG_FACTOR_2 in Appendix 3) reduces the problem of factoring over $\{K(a) \mid f(a)=0\}$ to the problem of factoring over $K$, and this can be applied repeatedly to reduce the problem to factoring over $K(X)$, and this was solved above.

The above remarks might lead one to believe that a system with "good" support for algebraic objects is sufficient for an algebraic-geometric system. That this is not completely true is demonstrated by the following example of the behaviour of algebraic expressions under transformations of the type commonly performed: $\sqrt{x^{2}-1} \rightarrow$ (under $x \rightarrow 1 / x$ ) $\sqrt{1 / x^{2}-1}=\sqrt{1-x^{2}} / x=\sqrt{x^{2}-1 / x} \rightarrow$ (under $x \rightarrow 1 / x$ in order to reverse the effect of the previous transformation) $-\sqrt{x^{2}-1}$, thus proving that $1=-1$. The solution to this difficulty is to have a separate basis for the algebraic objects in the system for each "value" of $x$, so that, in the above example, we would not express $\sqrt{1-x^{2}}$ as $\sqrt{x^{2}-1}$ since we have no reason to do so. Thus some form of programmer control over the system's handling of algebraic entities is required. This effect is not wholly destructive, however,

\# Note that we are not allowing $F$ to involve $Z$ at all. We require each algebraic to be determined, in terms of the previous ones (and hence in terms of $X$ ) by precisely one equation. If this restriction were to be relaxed, we would need to consider the whole question of Groebner Bases (see the discussion in Buchberger, 1979). 
since current algorithms for determining dependencies between algebraic expressions (Trager,1976) have complexity at least exponential in the number of algebraic expressions to be considered. Therefore the fact that we need not consider algebraic expressions at one value of $x$ when working at another can lead to an enormous reduction in complexity.

\section{Implementation Considerations}

Here we consider the practical implications of the previous paragraphs, and how the processes described are implemented in the program (see also Appendix 1 for more technical discussions of the changes made to REDUCE-2).

The most important remark is that the program deals only with square roots, i.e. an equation $F(X, Y)$ has to be of the form $Y^{2}-G(X)$. Since we have a multivariate representation of algebraic expressions, we can represent several square roots simultaneously, and we can represent nested square roots. The decision to impose this restriction was taken on purely practical grounds: all the problems of integration can be illustrated by examples expressed in terms of square roots, and code which is limited to square roots is significantly shorter ${ }^{*}$ and faster than more general code. Since the development of this program has severely strained the computing resources avalable, this consideration was extremely important.

Corresponding to each "basic-place" (this term is explained in the next section, but can be viewed as a value of $X$, with corresponding values of $Y, \ldots)$, we maintain a separate list of algebraic expressions which determine the field $\{K(X, Y, \ldots) \mid F(X, Y)=0 \ldots\}$, each expression determined by an equation irreducible over the extension defined by all its predecessors. Any time a new algebraic expression is considered, it is tested for independence with respect to the list corresponding to the correct basic-place. This mechanism

\footnotetext{
* The main reason for this is that a square root has precisely one conjugate, which can be obtained by negating the original expression, whereas a general algebraic expression can have several. Furthermore the conjugates of a general expression need not even be defined over the field in which the expression lies.
} 
avoids the difficulty described at the end of the previous section, and ensures that we do not consider algebraics for independence when this is not necessary.

We do not in fact work in terms of variables $X, Y, \ldots$; we work in terms of $X$ only, and $Y$ (where $\left.F(X, Y)=Y^{2}-G(X)=0\right)$ is represented by the expression SQRT $(\mathrm{G}(\mathrm{X})$ ), which is a REDUCE-2 kernel (Hearn,1973), i.e. it can be regarded as a separate variable for the purpose of forming polynomials and rational functions. The simplification rule

FOR ALL Z LET SQRT(Z)**2=Z;

is declared, and calls (in principle. The actual program has been optimised: see Appendix 1 item 4 for details) to the simplification routine are then written into the code as necessary. This process is unfortunately error-prone, and many of the bugs in the code have been caused by simplifying when I ought not to have done (i.e. working in $\{K(X, Y) \mid F(X, Y)=0\}$ when $I$ should have been in $K(X, Y))$ or vice versa.

The problem of needing to write simplification calls "sometimes" is one of the greatest problems with the use of REDUCE-2 for algebraic computations, and many other computer algebra systems have the same problems. One of the design aims of the SCRATCHPAD/370 system now under development at IBM Yorktown Heights (Jenks, 1979 and Davenport \& Jenks, 1980) is the elimination of this confusion by the provision of a much wider range of possible domains for computation.

\section{Algebraic Geometry}

We can view the relationship $F(X, Y)=0$ as the defining equation of an algebraic curve in the space $K(X, Y)$, and this will prove a very useful point of view in the rest of this work. For a view of Algebraic Curves and Algebraic Geometry at the level we will need, see (Fulton,1969) or (Seidenberg,1968). A completely algebraic approach can be found in (Chevalley,1951), while a hybrid approach is contained in the book by Eichler (1966). The algebraic curve could be viewed as a Riemann Surface with branches, but in fact the standard terminology of algebraic geometers is to talk about "places", a place being the same as a branch, although the standard definitions are in terms of local valuation rings. 
We speak of a place lying over a value $A$ of $X$ (possibly including infinity), or being centred at that value, to mean that the function $X-A$ (or $1 / X$ in the case of infinity) takes the value 0 at that place.

Such an algebraic curve can have multiple points, but corresponding to every algebraic curve (and to every finitely generated algebraic extension of $K(X)$ ) there is a "non-singular model" of the curve (see Fulton,1969 Chapter 7, Theorem $3 \&$ Corollary), which is obtained from the original curve by "blowing up" all the singularities on it. This picturesque terminology means that the point in the plane which was the multiple point has been replaced by a line, and the multiple point on the curve has been replaced by several simpler points, and if this process is repeated sufficiently often, we will end up with only simple points. Unfortunately this non-singular model is no longer a curve in the $X, Y$ plane, and it is hard to visualise or manipulate this curve directly. So this Theorem about the existence of non-singular models is in fact not of great computational use.

Geometrically, there are two possible types of behaviour at a multiple point, depending on whether or not the tangents are multiple. A point of multiplicity 2 with 2 separate tangents can be seen at the origin in $Y^{2}=X^{2}-X^{3}$, whereas $Y^{2}=X^{3}$ has a point of multiplicity 2 with one tangent at the origin. Of course it is possible for both types of behaviour to exist simultaneously at the same point, (e.g. a point of multiplicity 7 with 3 tangents), but this is not inherently more complicated. A point of multiplicity $n$ with $n$ distinct tangents to the curve at it is termed an ordinary multiple point. A much more useful transformation than the one described in the previous paragraph is one that transforms a plane curve to a birationally equivalent plane curve, all of whose multiple points are ordinary (see Fulton, 1969, chapter 7). In practice, it is normally sufficient to do this type of de-singularisation "locally", i.e. to transform a non-ordinary multiple point away if one finds that a particular multiple point is troublesome, rather than attempt to find all the multiple points and perform this transformation at all of them. These techniques, plus a recognition of ordinary multiple points, allow one to perform calculations on algebraic curves without being bedevilled by the potential singularities, although the code must always allow for the possibility of there being a singularity and make the necessary transformations. One other kind of transformation which we may frequently wish to make 
is the birational transformation, which is a map from a curve in $\left(x_{1}, \ldots, x_{n}\right)$ space to a curve in $\left(y_{1}, \ldots, y_{m}\right)$ space such that all $x_{i}$ are rational functions of the $y_{j}$ and vice versa. If there is a birational transformation from $A$ to $B$, we say that these are birationally equivalent.

Lemma $1 *$ Two curves are birationally equivalent iff their function fields are isomorphic.

\section{Puiseux Expansions}

A major tool in Algebraic Geometry is the theory of Puiseux Expansions. A Puiseux Expansion about a place is the equivalent of a Laurent Expansion about a point in ordinary function theory. Just as in conventional function theory we cannot express $\sqrt{X}$ as a Laurent Series (with integral exponents) in terms of $X$, so on $\left\{K(X, Y) \mid Y^{2}-X=0\right\}$ we cannot express $Y$ as a Puiseux expansion in terms of $X$ about $X=0$, even though we can express $X$ in terms of $Y$ about the same point. However, for any place centred at $A$, there is always some fractional power of $(X-A)$ ( or $1 / X$ in the case on infinity) such that all functions in $\{K(X, Y) \mid F(X, Y)=0\}$ can be expressed as Puiseux expansions in terms of it (see Chevalley, 1951, Chapter I, Theorem 2). Any function such that all functions can be expressed as Puiseux expansions in terms of it about a place, but such that no integral power of it will suffice, is termed a uniformising variable or local parameter. We shall frequently need to bear in mind the fact that $(X-A)$ or $1 / X$ need not be a local parameter. A place at which it is not a local parameter is said to be ramified, and the power of the local parameter which gives $(X-A)$ or $1 / X$ is said to be the ramification index $\$$ (and hence an unramified place has ramification index 1 ).

We define the order of a function at a place to be the index of the first non-zero term

* Fulton (1969) Proposition 12, p. 155.

$\$$ The terms "ramified" and "ramification index" are also used in algebraic number theory with somewhat different meanings, and we shall need these other meanings (which we shall define there) in Chapter 8 . It should always be clear from the context whether we are using the geometric or number-theoretic meanings. 
in its Puiseux expansion about this place divided by the ramification index of that local parameter. This is clearly independent of the local parameter chosen.

\section{A Data Structure for Places}

We require some form of data structure to represent the mathematical concept of a place in our program. The overall format of the data structure $I$ have chosen is that of a LISP substitution list, i.e. a list of pairs (old value . new value).

The first pair in the list determines the value of the variable of integration (which we shall assume to be $X$ for the purpose of all the examples) over which the place lies. If the place lies over $X=0$ the first pair is (X.X), if the place lies over the point at infinity, the first pair is (X QUOTIENT $1 \mathrm{X}$ ), and if it lies over the point $X=a$, the first pair is (X PLUS $X-a)$. After this substitution has been performed, the variable $X$ now takes the value 0 at the point over which the place lies.

However $X$ need not be a local parameter. The second element of the list is a substitution pair designed to rectify this problem (if it exists) and is (X EXPT X n), where $X^{1 / n}$ is a local parameter at the place in question. If $X$ was already a local parameter, this component is not present. This part (if present) and the previous part constitute a basic-place, i.e. an expression for a local parameter at a point such that all that is required to convert it into a description of a place is some way of determining which place over that point is intended.

The remainder of the list is designed to discriminate between the various places lying over one point. This can be much more difficult than it seems, for the places are in some sense indistinguishable, all being roots of the algebraic equation defining the curve. A purely arbitrary decision can be made, calling one of the roots ${ }^{*}$ of $Y^{2}=X^{2}-1$, for example, SQRT $\left(X^{* * 2}-1\right)$, and the other one $-\operatorname{SQRT}\left(X^{* * 2-1)}\right.$. Then this part of the list

* In the case where $F(X, Y)$ is not of the form $Y^{2}-G(X)$, but is of degree $n$ in $Y$, we will have $n$ possibilities, corresponding to the conjugates of $Y$. 
contains substitution pairs of the form (Y.Y) or (Y MINUS Y), where $Y$ is an expression of the form SQRT(polynomial in $X$ ). $Y$ is expressed as if the transformations in the first and second part of the list had been conducted, e.g. the two places of $Y^{2}=X^{4}+X^{3}+1$ lying over infinity are ((X QUOTIENT $1 \mathrm{X})$ (expr.expr)) and ((X QUOTIENT $1 \mathrm{X})$ (expr MINUS expr)), where expr is SQRT( $1+X+X * 4)$.

With this data structure, the evaluation of functions at places ought to be straightforward, in that one should substitute $X=0$ in the result of substituting the expression for the place into the function. Unfortunately, this frequently gives $0 / 0$ as the answer.

The immediate answer to this is to use l'Hopital's rule for the evaluation of indeterminate limits, viz. that $\lim F(X) / G(X)=\lim F^{\prime}(X) / G^{\prime}(X)$ if $G(0)=0$. While this rule is extremely useful to the mathematician, there is no guarantee that $F^{\prime}(X) / G^{\prime}(X)$ is easier to evaluate than $F(X) / G(X)$, and it may well be harder - the reader may care to consider

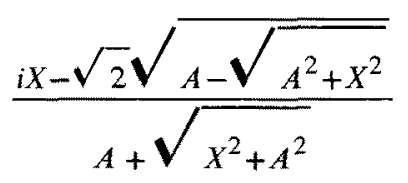

where $F^{\prime}$ and $G^{\prime}$ are both more complex than $F$ and $G$. The answer to this problem is in fact to calculate such values as the coefficient of $X^{0}$ in Puiseux expansions. Harrington, in his work (Harrington, 1979a) on the evaluation of limits, has a variety of applications of l'Hopital's rule, but ends up admitting that Taylor series (equivalent to Puiseux expansions) may be necessary for the evaluation of some limits.

\section{Computation of Puiseux Expansions}

The computation of Puiseux expansions is a problem very similar to the computation of Taylor (or, more strictly, Laurent) Series. Before this problem is dismissed as totally trivial, it is worth considering the question "What is the coefficient of $X^{0}$ in 
$(1+Y) /(1-Y)$, where $Y^{2}=X^{2}+1$ ?" \#. A straightforward computation will give us $2 / 0$, and in order to obtain the correct answer we have to discover the coefficient of $X^{2}$ in $1+Y$ and $1-Y$. Also, having computed a power series as far as the $X^{4}$ term, say, we may need the $X^{5}$ term, and it would be wasteful to recompute the entire series in order to obtain the next term.

The best technique for expanding power series in this way is the "incomplete but exact" approach of Norman (1975). Norman summarises the design by saying that his package "gives the impression that it is computing with full rather than truncated power series. Calculations are only performed when an attempt is made to display or use the results, and so no unnecessary work is ever done".

Norman was able to use the SCRATCHPAD system of rules and values in order to produce an extremely neat implementation. We do not have this mechanism available in REDUCE-2, but we can perform equivalent functions. The representation of a Puiseux Expansion is a data structure with two components: a list of already computed terms, and a method of evaluating terms in it, which will be an operator $\left(+,-,{ }^{*}, /, S Q R T\right)$ and a list of arguments, which are themselves Puiseux Expansions of components of the original expression.

\section{Divisors}

We can construct the free Abelian group on all the places of an algebraic curve, i.e. the set of all finite collections of places with integer multiplicities. An element of this group is termed a divisor. This group can be written either multiplicatively or additively, and the literature has both. We will normally find it convenient to write it multiplicatively. The sum of all the multiplicities is termed the degree of the divisor. One way of regarding the multiplicities in a divisor is to say that they tell you whether this place is a zero (of the multiplicity specified in the divisor) or a pole (with the multiplicity specified in the divisor,

\# The answer is in fact -2 . 
but ignoring the - sign) of a specified function. In this case the divisor $D$ is termed the divisor of the function $f$ specified, and we write $D=(f)$. Every function except the zero function has a divisor, and the mapping from the multiplicative group of non-zero functions into the group of divisors is in fact a group homomorphism. The kernel of this homomorphism is precisely the set of constant functions (i.e. the non-zero elements of $K$ ). The divisor of a function always has degree 0 (Chevalley, 1951 Chapter 1, Theorem 5 ), but the converse is not always true. In the special case of $K(X)$ it is true, for one multiplies together the $(X-A)^{N}$ for all finite places lying over $A$ with multiplicity $N$, and this necessarily has the correct multiplicity over infinity. A divisor with no negative multiplicities is said to be effective.

In general there are three possibilities for a divisor of degree 0 : it can be the divisor of a function in $\{K(X, Y) \mid F(X, Y)=0\}$, when we say that the divisor is principal or linearly equivalent to zero; there can be a power of it which is linearly equivalent to 0 , when we say that the divisor is rationally equivalent to 0 , or a torsion divisor; or there can be no power of it which is the divisor of a function, when we say that the divisor is (rationally) inequivalent to 0 . If the divisor is rationally equivalent to 0 , then the power to which it must be raised to make it linearly equivalent to 0 is termed the order of the divisor.

In the case of $K(X)$, not only are all divisors of degree .0 linearly equivalent to 0 , but, as shown above, it is trivial to find the function which corresponds to a divisor of degree 0 . In general this is not true, and the breakthrough in finding such a function, if the divisor is linearly equivalent to 0 , was the work of Coates (1970), described in the next chapter.

\section{Differentials}

Another useful concept in Algebraic Geometry is that of the differential, which can be thought of as an expression of the form $f(X) \mathrm{d} X$, even though a rigorous definition has to be much more abstract (see Fulton,1969, pp. 203-5). We regard differentials as being the same if the ordinary rules of schoolboy calculus would make them so (see Fulton,1969 again for a precise description), e.g. $\mathrm{d}\left(X^{2}\right)=2 X \mathrm{~d} X$. We can make the differentials into a 
$\{K(X, Y) \mid F(X, Y)=0\}$ module in the obvious way, e.g. $c \mathrm{~d} X=\mathrm{d}(c X)$ for $c$ in $K$. The space of all differentials is a 1-dimensional vector space over $\{K(X, Y) \mid F(X, Y)=0\}$, and $\mathrm{d} X$ is a basis for it.

If $t$ is a uniformising parameter about a place $P$, then any differential can be written as $f \mathrm{~d} t$ for some $f$ in $\{K(X, Y) \mid F(X, Y)=0\}$. Define the order of the differential at $P$ to be the order of $f$ at $P$ (this is well-defined by Fulton, 1969 Chapter 8, Prop. 7). Hence we can define the divisor of a differential in the same way as we defined the divisor of a function earlier. Note that the two are different ideas: the divisor of $1 / X$ is a pole of order 1 at 0 and a zero of order 1 at infinity, while the divisor of $1 / X \mathrm{~d} X$ is a pole of order 1 at 0 and a pole of order 1 at infinity (because $t=1 / X$ is a uniformising parameter there, and $1 / X \mathrm{~d} X=t(\mathrm{~d} X / \mathrm{d} t) \mathrm{d} t=-1 / t \mathrm{~d} t)$.

A differential is said to be of the first kind if it has no poles. An example of this is $1 / Y \mathrm{~d} X$ when $F(X, Y)=Y^{2}-\left(X^{3}+1\right)$. Differentials of the first kind form a vector space over $\mathrm{K}$, and the dimension of this space is termed the genus or deficiency of the curve, often denoted by $g$. The genus is a parameter of the curve of great importance in Algebraic Geometry. In particular, a curve of genus 0 can be transformed into a line by means of a rationalising substitution. This is the justification for the use of trigonometric substitutions to integrate functions defined over curves of genus 0 (of which $F(X, Y)=Y^{2}-\left(1-X^{2}\right)$ is the simplest example), because if $X$ is $\cos t$, then $Y$ is $\sin t$, and both of these are rational functions of $\tan t / 2$, which is an actual rationalising parameter. A curve of genus 1 is said to be an elliptic curve. We will discuss these curves in greater detail in Chapters 5 and 7.

As in classical function theory, we can define the residue of a differential at a place. There are a variety of abstract definitions, but for our purposes the definition which says that it is the coefficient of $t^{-1}$ in an expansion of the differential in terms of a uniformising parameter $t$ is sufficient. If the residue of a differential is non-zero then that place must be a pole of the differential, so a differential can only have a finite number of non-zero residues. A differential, all of whose residues are zero, is said to be of the second kind. Clearly every differential of the first kind is of the second kind also. The differentials of 
the second kind form a $2 g$ dimensional vector space over the space of exact differentials. A differential is said to be of the third kind if all its poles have order 1. Differentials which are of both the second and the third kind must be of the first kind.

The representation of a differential is precisely the same as that of a function: however the rules for transforming and evaluating it are not the same. Because a differential is $f \mathrm{~d} t$ for some function $f$ and uniformising parameter $t$, when we wish to evaluate the differential at a place we must transform the function $f$ (as described in the section "A Data Structure for Places" above), and also multiply by $\mathrm{d} t / \mathrm{d} t^{\prime}$, where $t^{\prime}$ is a uniformising parameter at the new place. 